\title{
Performance Analysis of Filter based on Functional Link Artificial Neural Network
}

\author{
Suchitra Sarangi \\ Department of E\& TC \\ C.V.Raman College Of Engg \\ Bhubaneswar,India
}

\author{
Shubhendu Kumar Sarangi \\ Department of EIE \\ ITER, SOA UNIVERSITY \\ Bhubaneswar, India
}

\author{
Sunita Sarangi \\ Department of EIE \\ ITER, SOA UNIVERSITY \\ Bhubaneswar, India
}

\begin{abstract}
Image restoration is an important part of image processing. There are several techniques exist for image recovery. This paper presents a functional link artificial neural network based technique for image restoration which has the capacity of reducing the Gaussian noise present in an image. Then a comparison has been carried out between the proposed filter $\&$ the other existing filters. Finally, some conclusion $\&$ future work lines are presented.
\end{abstract}

\section{Keywords}

Image restoration, noise \& its parameters, different filters, functional link artificial neural network.

\section{INTRODUCTION}

There is a chance of image corrupted with noise during the acquisition, transmission, storage \& recovery processes. Image transmitted through channels from source to destination gets noisy due to the interference in the channel. Also the images that are transmitted through wireless network might be corrupted because of lightning or any other atmospheric disturbances.

When an image encountered with noise, the pixel values of the image changes depending upon the characteristics of noise. If we analyzed, there are several solutions for the noisy image like median filter is mostly used for removal of salt \& pepper noise while mean filtering is used for the removal of Gaussian noise present in an image etc.

This paper presents a method for image restoration, which is based upon the theory of functional link ANN (FLANN) that was proposed by Pao [5], where the hidden layer is eliminated by expanding the input pattern by choosing suitable functional expansions.

In the proposed method chebyshev polynomials used [14] as the functional expansion. The experimental results show that this modified approach is better than the original non linear adaptive filter[17]designed using feed forward neural network. The remaining of this paper is organized as follows: section 2 discusses about the related works, the way in which the filter is applied to a noisy image is described in Section 3. Section 4 describes the result. Section 5 gives the conclusion about the study.

\section{RELATED WORKS}

Hamza et al. [8] introduced a nonlinear filtering technique based on the theory of robust estimation. The proposed method combine different filtering methods such as mean, relaxed median, and the LogCauchy filter. This method gives better result for mixed noise as compared to other discussed methods.

Bilcu et al. [10] introduced a new sigma filter for noise reduction in images that has improved performance in terms of MES \& provides better fine details than the standard sigma filter. The proposed method first decomposes the input image in four components that are independently processed using a standard sigma filter. The output image is reconstructed from the filtered components.

Gallo et al. [13] proposed a method to deal with the noise problem which is based on neural learning for blind image restoration. A solution to this is local estimation of the restored image based on gradient descent strategies able to estimate both bluring function \& regularized terms adaptively. The drawbacks of this method is it's computational complexities \& the quality of restoration.

Roomi et al. [9] have presented a no reference blur metric based iterative edge preserving filtering technique which is selective on noisy \& edge pixels. From the experimental result analysis it is clear that that this filter is better than the state of art filters and gives better peak signal to noise ratio (PSNR). It is also useful to reduced the blur and preserves the edges of the images at higher noise levels.

Kervam et al. [12] proposed a novel image restoration approach for poisson noise reduction \& discontinuities preservation in images. It's advantage is there is no need of hidden parameters need to be precisely adjusted as in many other methods \& the algorithm can be parallelized.

Portilla et al. [7] describes a method for noise removal from digital images. This method is based on the theory of statistical model of the coefficients of an over complete multiscale oriented basis. The proposed model captures correlations induced by the over complete representation as well as correlations inherent in the underlying image. This method can reduced the Gaussian noise of arbitrary power spectral density.

Corbalan et al. [17] introduced a method based on feedforward neural network, which has the ability to recover the image that is corrupted by Gaussian noise having zero mean $\& 0.01$ variance. The network has three layers and to train the network back propagation algorithm is used. It uses an eight component vector which is given as the input to the network. This method can also be used to the x-ray images. 


\section{PROPOSED WORK:}

\subsection{Non-Linear Adaptive Filter Based on \\ FLANN}

The proposed method, i.e. the FLANN structure, is a single layer artificial neural network structure with the ability of taking complex decision regions from nonlinear decision boundaries. In a FLANN there is no hidden layer. The nonlinearity of the netwok improved by choosing appropriate functional expansions. Here the functional expansion block comprises of Chebyshev polynomials.

Here four additional matrices discussed in [17] are consider with I' as the noisy image are considered i.e.

$$
\begin{aligned}
& A=\left(\begin{array}{ll}
1 & -1
\end{array}\right) \otimes I^{\prime}, \\
& B=\left(\begin{array}{c}
1 \\
-1
\end{array}\right) \otimes I^{\prime}, \\
& C=\left(\begin{array}{cc}
-1 & 0 \\
0 & 1
\end{array}\right) \otimes I^{\prime}, \\
& D=\left(\begin{array}{cc}
0 & -1 \\
1 & 0
\end{array}\right) \otimes I^{\prime},
\end{aligned}
$$

The eight-component vector discussed in [17] which is the input to the network is specified as:

$$
\text { input }(i, j)=\left(\begin{array}{c}
A(i, j-1) \\
A(i, j) \\
B(i-1, j) \\
B(i, j) \\
C(i-1, j-1) \\
C(i, j) \\
D(i-1, j) \\
D(i, j-1)
\end{array}\right)
$$

Then the 8 inputs are expanded each of according to Chebyshev expansion. The tanh(.) activation function is used at the output. The difference between the original image and the noisy image is given as the target to the network. The LMS algorithm is used to train the FLANN becomes simple and has a faster convergence due to its single layer architecture.

The chebyshev polynomials are easier to compute than the trigonometry polynomials. The first few Chebyshev polynomials are given by

$$
\begin{aligned}
& T_{0}(x)=1.0 \\
& T_{1}(x)=x \\
& T_{2}(x)=2 x^{2}-1
\end{aligned}
$$

The higher order Chebyshev polynomials may be generated by the recursive formula given by:

$$
T_{n+1}(x)=2 x T_{n}(x)-T_{n-1}(x)
$$

\subsection{Different noise Parameters}

The peak signal-to-noise ratio (PSNR) and mean square error (MSE) of the filters can be calculated using the following formula:

$\mathbf{M S E}==\frac{\sum_{m=1}^{M} \sum_{n=1}^{N}(X(m, n)-Y(m, n))^{2}}{M x N}$

Where $\mathrm{X}(\mathrm{m}, \mathrm{n})$ and $\mathrm{Y}(\mathrm{m}, \mathrm{n})$ are the original and the noisy image respectively.

\section{PSNR $=10 \log _{10}(255)^{2} / \mathrm{MSE}$}

\section{RESULTS}

\subsection{Comparative Study of various Filter}

Several images were taken and corrupted with Gaussian noise having zero mean $\& 0.01$ variance. Then the corrupted images were passed through the network and a comparison was made with different filters outputs. The size of the images are 256x256 pixels.

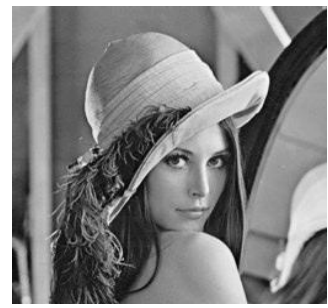

(a)

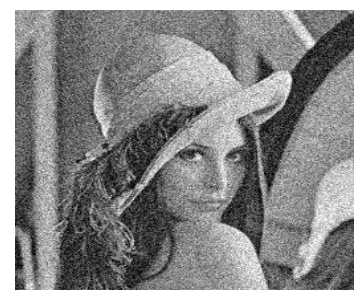

(b)

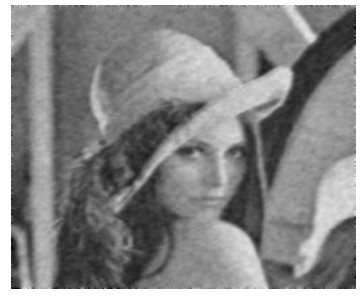

(c) 


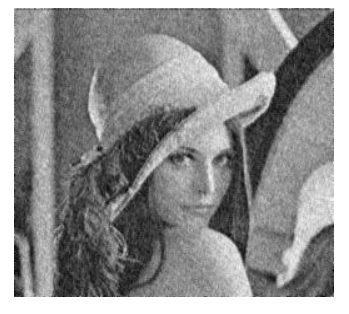

(d)

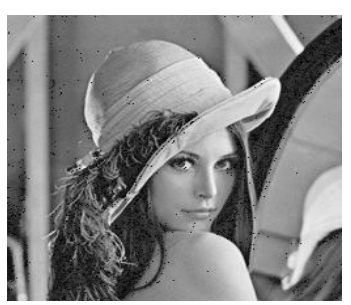

(e)

Figure 4.1: (a) original image, (b) noisy image, (c) mean filter output, (d) Network output, (e) FLANN output

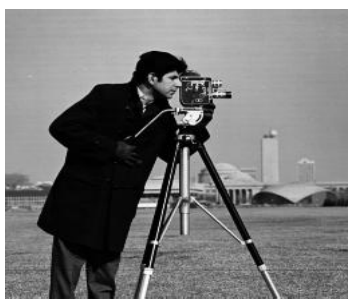

(a)

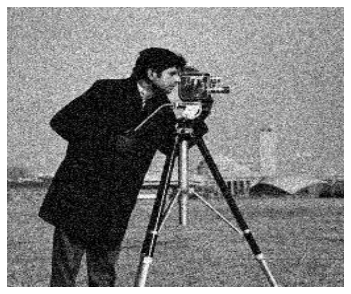

(b)

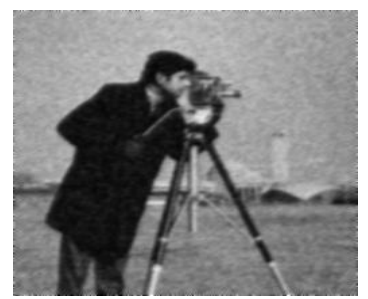

(c)

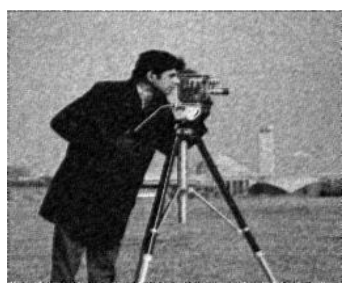

(d)

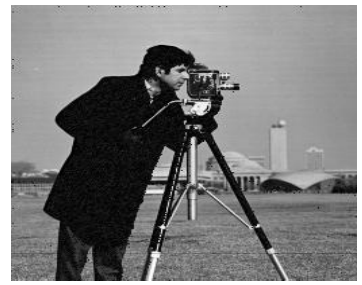

(e)

Figure 4.2: (a) original image, (b) noisy image, (c) mean filter output, (d) Network output, (e) FLANN output

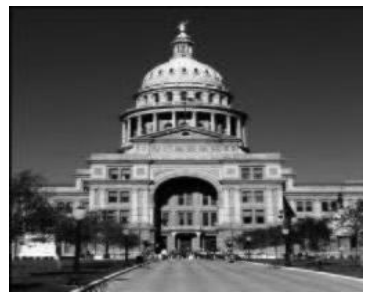

(a)

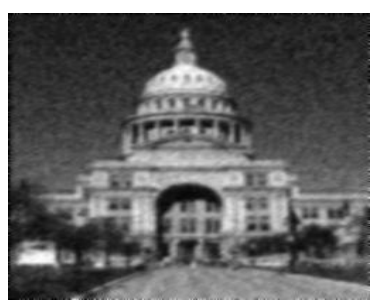

(b)

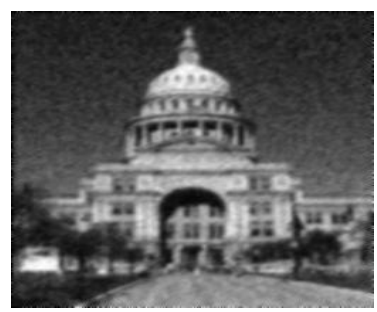

(c) 


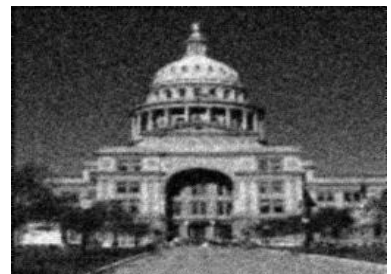

(d)

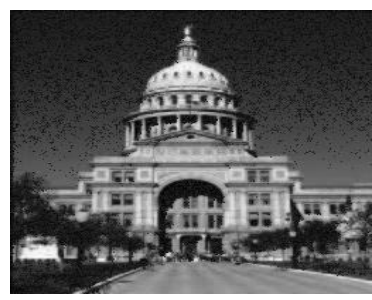

(e)

Figure 4.3: (a) original image, (b) noisy image, (c) mean filter output, (d) Network output, (e) FLANN output

Table:1 PSNR value for Gaussian noise

\begin{tabular}{|l|l|l|l|l|}
\hline Image & Noisy & MEAN & NN & FLANN \\
\hline Lena & 20.05 & 25.90 & 27.37 & 29.08 \\
\hline Cameraman & 20.38 & 23.15 & 26.54 & 29.73 \\
\hline Iqbal_0022 & 20.29 & 25.33 & 26.66 & 28.00 \\
\hline
\end{tabular}

Table:2 Time Duration for Gaussian noise in sec

\begin{tabular}{|l|l|l|l|}
\hline Image & MEAN & NN & FLANN \\
\hline Lena & 1.46 & 21.06 & 9.11 \\
\hline Cameraman & 1.06 & 21.66 & 9.70 \\
\hline Iqbal_0022 & 0.82 & 21.21 & 9.14 \\
\hline
\end{tabular}

\subsection{Performance of FLANN at higher noise}

\section{Level}

Several test images were taken and corrupted with Gaussian noise with zero mean \& variance ranging from 0.1 to 0.9 to know the performance of the filter at higher noise levels.. The size of the images are $256 \times 256$ pixels. The result of noisy \& denoised images are shown in fig 4.3

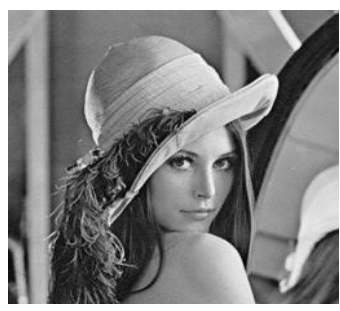

(a) Original image

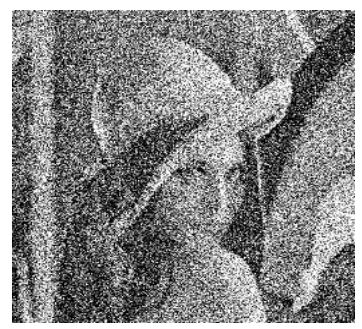

(b) $10 \%$ Noisy image

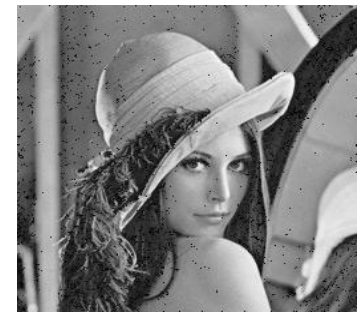

(c) Denoised image

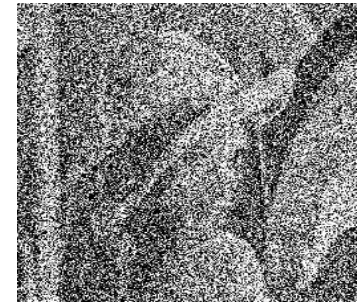

(d) $30 \%$ Noisy image

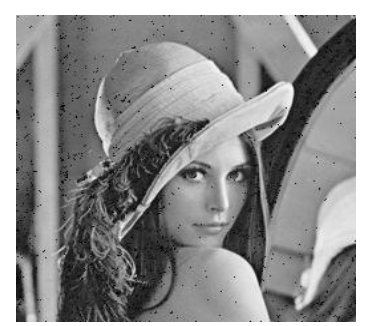

(e) Denoised image 


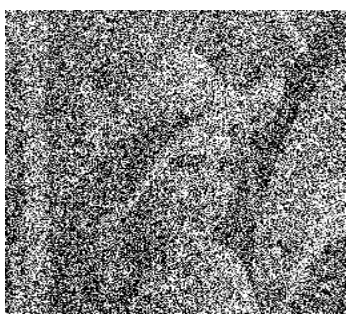

(f) $60 \%$ Noisy image

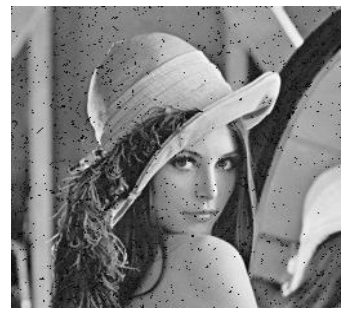

(g) Denoised image

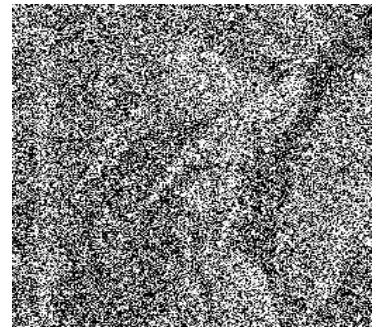

(h) $90 \%$ Noisy image

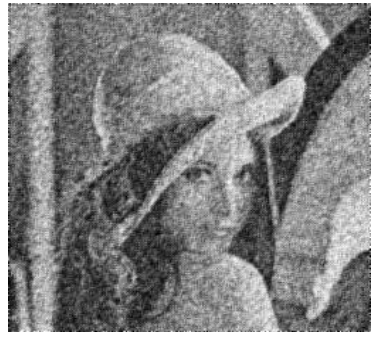

(i) Denoised image

Fig 4.4(a-i) Noisy \& Denoised lena.jpg image at noise variance $0.1,0.3,0.6 \& 0.9$ respectively.
Table: 1 PSNR of noisy \& denoised lena.jpg image using proposed method

\begin{tabular}{|r|r|r|}
\hline Variance & Noisy image & Denoised image \\
\hline 0.1 & 11.32 & 25.15 \\
\hline 0.2 & 9.32 & 24.54 \\
\hline 0.3 & 8.68 & 23.33 \\
\hline 0.4 & 8.19 & 21.84 \\
\hline 0.5 & 7.89 & 21.03 \\
\hline 0.6 & 7.64 & 20.87 \\
\hline 0.7 & 7.46 & 20.03 \\
\hline 0.8 & 7.33 & 19.56 \\
\hline 0.9 & 7.19 & 19.11 \\
\hline
\end{tabular}

\section{CONCLUSION}

A nonlinear filter based on FLANN has been proposed for the removal of Gaussian noise. From the simulation results it is clear that Functional link Artificial Neural Network having Chebyshev expansion works better than mean filter as well as the network filter for Gaussian noise separation from corrupted images. The time taken by FLANN is also less as compared to ANN \& it has less computational complexity since there is no hidden layer.

The FLANN may be used for online image processing application due to its less computational requirement and satisfactory performance.

\section{REFERENCES}

[1] Rafael C. Gonzalez and Richard E. Woods, "Digital Image Processing", Pearson Education (Singapore) Pte Ltd, 2004.

[2] Anil K. Jain, "Fundamentals of Digital Image Processing”, Prentice Hall of Inc, 1998.

[3] B. Chandra, D. Dutta Majumder, "Digital Image Processing and Analysis", Prentice Hall of India Private Limited, New Delhi 2000.

[4] J. C Patra., R. N. Pal and B. N.Chatterji, "Identification of non-linear dynamic systems using functional link artificial neural networks," IEEE Trans. on Neural Networks, vol. 29, no. 2, pp. 254-262, 1999.

[5] Y.H .Pao .Adaptive Pattern Recognition and neural networks. Reading .MA addison-Wesley.1989.

[6] Naira Hovakimyan, Flavio Nardi, Anthony Calise, and Nakwan Kim, "Adaptive output feedback control of uncertain nonlinear systems using single-hidden-layer neural networks," IEEE Trans. on Neural Networks, vol. 13 , no. 6 , pp. 1420-1431, 2002.

[7] J.Portilla, V.Strela, M.J.Wainwright, .P.Simoncelli,"Image denoising using scale mixture of 
Gaussian in the wavelet domain", IEEE Transactions on Image Processing,vol.12, no. 11 pp.1338-1351, November 2003

[8] A.B.Hamza, H.Krim,'Image Denoising: A Nonlinear Robust Statistical Approach",IEEE Tranactions on Signal Processing,vol.49, no.12,December 2001.

[9] S.Md.M.Roomi, T.Pandy Maheswari, V.A.Kumar,"A Detail Preserving Filter for Impulse Noise Detection and Removal”, ICGST-GVIP Journal, Volume 7, Issue 3, November 2007

[10] Z.Long, N.H.Younan,"Denoising Of Images With Multiplicative Noise Corruption",Mississippi State University,Starkville, MS 39759,USA.

[11] C.Kervrann, A.Trubuil," An Adaptive Window Approach For Poisson Noise Reduction And Structure Preserving In Confocal Microscopy",

[12] I.Gallo, E.Binaghi and A.Macchi,"Adaptive Image Restoration Using A Local Neural Approach",Italy.

[13] Jagdish C.Patra, Alex C.Kot,Non-linear Dynamic System
Identification Using Chebyshev Functional Link Artificial Neural Networks,IEEE Transaction On Systems,Man and Cyberntics, vol.32.no.4 August 2002

[14] J.Astola and P.Kuosmanen,Fundamentals of Non-linear Digital Filtering.Boca Raton, FL:CRC,1997

[15] Guez Allon, James L. Ellbert and Moshe Kam, "Neural networks architecture for control," IEEE Control System magazine, pp. 22-25,1998.

[16] L.Corbalan, G.Osella Massa, C.Russo, L.Lanzarini, A.De Giusti,"image recovery using a new nonlinear adaptive filter based on neural networks," jounral of computing mation technology-cit 14, 2006, 4, pp315-320

[17] A. Sierra, J. A. Macias and F. Corbacho, "Evolution of functional link networks," IEEE Trans. on Evolutionary Computation, vol. 5, no. 1, pp 54-65, 2001

[18] A. K Goel. and S Bhanot, "Modelling of continually stirred tank heater with ANNs using successive overrelaxation backpropagation algorithm," ASCC'02, Proc of the Asian Control Conf., (Singapore), pp.614-617, 2002 . 